\title{
Optimization Natural Gas Pumping Units Operation by the Economic Criterion in the Conditions of Uncertainty
}

\author{
Gorbiychuk Mikhail Ivanovych \\ Ivano-Frankivsk National Technical \\ University of Oil and Gas \\ Ivano-Frankivsk, Ukraine \\ https://orcid.org/0000-0002-8586-1883
}

\author{
Bila Olga Tarasivna \\ Ivano-Frankivsk National Technical \\ University of Oil and Gas \\ Ivano-Frankivsk, Ukraine \\ https://orcid.org/0000-0003-2245-7434 \\ Humeniuk Taras Volodimirovych*
Ivano-Frankivsk National Technical
University of Oil and Gas
Ivano-Frankivsk, Ukraine
https://orcid.org/0000-0003-2610-2550
}

\author{
Slabinoha Marian Ostapovych \\ Ivano-Frankivsk National Technical \\ University of Oil and Gas \\ Ivano-Frankivsk, Ukraine \\ https://orcid.org/0000-0002-7296-0356
}

\begin{abstract}
The article deals with the method of determining the number of parallel operating units and the number of the rotor rotations for each supercharger in the group by the economical criterion, taking into account the cost of fuel gas and the power of nitrogen oxides into the atmosphere. At the same time, limitations on technological parameters and conditions of uncertainty are taken into account, which are caused by inaccuracies of measurements and influence of uncontrolled parameters on the process of natural gas compression.
\end{abstract}

Keywords—gas pumping units, natural gas, technical conditions, artificial intelligence, optimization.

\section{INTRODUCTION}

At the present stage of operation of the Ukraine gas transmission system (GTS), one of the important tasks is to save resources spent on production and technological needs and to reduce harmful emissions into the environment.

This can be achieved both by modernizing the existing fleet of gas-pumping units and by introducing modern automated natural gas transportation control systems.

Thus, the modernization plan of the GTS developed by Ukrtransgas [1] envisages the reconstruction of four compressor stations: Yagotin, Dykanka, Romny and a compressor station at the underground gas storage facility KC 4 Bilche-Volytsya. More than 7 billion UAH are planned to be invested in the reconstruction of the compression stations in 2018-2027.

Another way, less costly, is the introduction of modern automated natural gas transportation control systems, which should solve not only the problems of protection, control and stabilization of technological parameters, but also the optimal control of the operation of the compressor stations.

Thus, the purpose of the work is to develop methods and algorithmic support for a computer system for the compression of natural gas by the criterion, which ensures the saving of fuel gas taking into account harmful emissions into the environment in the conditions of uncertainty arising from the measurement of technological parameters and inaccuracies in the processing of information on technological process.

\section{MAIN RESEARCH}

For gas transportation by pipelines there are used gas pumping units (GPU), which are placed at the compressor stations. GPUs include natural gas centrifugal blowers (CB) with electric or gas turbine drive. JSC Ukrtransgaz operates 702 units of which 448 are gas turbine driven, accounting for $63.8 \%$ of their total. Ukrtransgas' natural gas costs for production and technological needs in 2018 amounted to 1.9 billion cubic meters. [1], most of which is burned in the combustion chambers of the GPUs in order to ensure the operation of the gas turbine drive. This part of gas is called fuel gas.

One of the most promising areas for reducing fuel gas consumption is to optimally operate the GPUs. It is necessary to take into account the technical condition of the working GPUs.

The technical states of the combustion chamber, oil system, thrust bearings and flowing part of the $\mathrm{CB}$ are evaluated. For each unit of the GPU, the characteristics are monitored and monitored over a period of time. Such features form a set of features, which in the theory of pattern recognition is called the set of images [2]. With the use of neural network technology, many images are divided into classes. Each class is assigned a specific rij rating, where i is the node number; $j$ is the GPU number. Then the rating of the $j$-th GPU is calculated by the following formula: $R_{j}=\sum_{i=1}^{n} r_{i j}$, where $n$ - the number of GPU units for which their technical condition is assessed. For values $R_{j}$ the technical condition assessment of GPU is done

$$
k_{j}=R_{j} / \sum_{j=1}^{m} R_{j},
$$


where $m$ - the number of GPUs installed at the compressor station.

Due to the reduction of natural gas transportation volumes, the compressor stations in Ukraine have excess capacity. Therefore, the problem arises of the optimal load distribution between the parallel working GPA.

Compressor stations where gas turbine-driven GPAs are installed are significant environmental pollutants. Particularly dangerous are emissions of nitrogen oxides [3]. Therefore, the optimality criterion takes into account not only the cost of the fuel gas but also the amount of the environmental fine for nitrous oxide emissions [4].

$$
\min : R(\bar{N})=c \sum_{i=1}^{L} N_{i} G_{i}+c_{f} \sum_{i=1}^{L} N_{i} E_{p, i}
$$

where $c$ - cost of fuel gas consumed, $U A H / k g ; N_{i}$ - number of GPUs in each of $L$ groups; $G_{i}$ - consumption of fuel gas by $i$-th GPU in the group consisting of $N_{i} \mathrm{CBs}, \mathrm{kg} / \mathrm{s} ; E_{p, i}$ capacity of emissions of nitrogen oxides by the $i$-th GPA, $\mathrm{kg} / \mathrm{s} ; c_{f}$ - amount of environmental tax, $U A H / \mathrm{kg}$.

The optimality criterion (1) should be minimized by the constraints defined by the natural gas transport task for the CS

$$
\sum_{i=1}^{L} q_{i} k_{i} N_{i}=Q_{0}
$$

where $q_{i}$ - the productivity of the $i$-th supercharger in the group $k_{i}$ - generalized coefficient of technical condition of the $i$-th supercharger in the group; $Q_{0}$ - the planned task of the compressor station for pumping gas is assigned to a unit of time.

Every GPU group has certain value of $M_{i}$ GPUs, which causes the resctrictions

$$
N_{i} \leq M_{i}, i=\overline{1, L} .
$$

In addition, the following obvious limitations must be met:

$$
N_{i} \geq 0, i=\overline{1, L} \text {. }
$$

Values $E_{p, i}$ i $q_{i}$, which appear in expressions (2) and (3), are functions of technological parameters. Dependencies (2) and (3) have rather complex functional dependencies, analytical expressions for which are unknown to date. Therefore, empirical models are used to describe them. A method based on the theory of genetic algorithms was used to construct empirical models. The result is a model of optimal complexity in a given class of models [4]. Generally, the model is chosen as a polynomial of a certain degree. GPUs, as objects of management, are externally influenced by the inaccuracy of measuring technological parameters. In addition, technological parameters such as supercharger performance and fuel gas consumption are measured indirectly. Emissions of nitrogen oxides are recorded with a different step of discretion compared to other technological parameters. All this gives grounds to consider $G_{i}$, s well as the technological parameters that determine the values of $E_{p, i}$ by fuzzy quantities. Function $E_{p, i}$ technological parameters as the $P_{a c}$ pressure and the $T_{a c}$ temperature at the outlet of the axial compressor, the inlet temperature of the high pressure turbine $T_{h t}$ and the fuel gas consumption $G$.

Dependency $E_{p, i}$ as a function of technological parameters is synthesized as a polynomial

$$
E_{p}(\overline{\mathrm{a}}, \overline{\mathrm{x}})=\sum_{i=0}^{M-1} a_{i} \prod_{j=1}^{m} x_{j}^{\varphi_{j i}},
$$

where $a_{i}$ - polynomial coefficients having the dimension of the nitrogen oxide emission power; $x_{j}$ - values that are reduced to a dimensionless form by the formula

$$
x_{j}=\left(X_{j}-X_{j, \text { min }}\right) /\left(X_{j, \text { max }}-X_{j, \text { min }}\right) \text {, }
$$

$x_{j}$ - technological parameters in dimensionless form that determine the value of the value $E_{p, i} ; X_{j}-j$-th technological parameter in units of measure; $X_{1}=P_{a c}, X_{2}=T_{a c}, X_{3}=T_{h t}$, $X_{4}=G ; \sum_{j=1}^{m} \varphi_{j i} \leq n_{\varphi} ; m$ - number of functional dependency arguments $(6)(m=4)$.

Powers of polynom $\varphi_{i j}, j=\overline{0, M-1}, i=\overline{1, m}$ form the matrix $\Phi$ with dimensions $M \times n$ with the sum of the rows not exceeding $n_{\varphi}$.

The number of members of the polynomial (6) can be determined by the following formula: $M=(m+n)$ ! / $(m ! n !)$.

The assumption [4] is that fuzzy quantities $x_{j}$, which are arguments of functional dependence $E_{p, i}$, have triangular membership functions $\mu\left(x_{k}\right)$.

After switching to the dimensionless formula (7) the values of the optimality criterion (2) will take the following form:

$$
\begin{aligned}
& \min : R(\bar{N})=\sum_{i=1}^{L}\left(c N_{i} \pi_{\mathrm{G}, i} x_{\mathrm{G}, i}+c_{f} N_{i} E_{p, i}\right)+ \\
& +c \sum_{i=1}^{L} N_{i} G_{i, \min },
\end{aligned}
$$

where $\pi_{G, i}=G_{i, \max }-G_{i, \min } ; x_{G, i}$ - is calculated using formula (7) by $X_{4}=G$.

The membership function $\mu\left(x_{j}\right)$ is an isosceles triangle whose height is unity and the base has a width $\Delta_{j}$. Since the membership function $\mu\left(x_{j}\right)$ is described by lump-continuous functions, it is inconvenient for practical use. Therefore, in [5] it is proposed to approximate the dependence $\mu\left(x_{k}\right)$ by the Gaussian membership function. If we take $\mu\left(x_{j}\right)=1 / 2$, where $x_{j}=x_{j, \text { min }}+\Delta_{j} / 2, x_{j, \text { min }}$ - is the minimum value of the $k$ th technological parameter, then as a result of the approximation we obtain the following Gaussian function:

$$
\hat{\mu}\left(x_{j}\right)=\exp \left(-\left(x_{j}-x_{j}^{(0)}\right)^{2} /\left(2 \sigma_{j}^{2}\right)\right),
$$


where $\mu\left(x_{j}^{(0)}\right)=1 ; x_{j}^{(0)}$ - modal value of fuzzy value $x_{j}$; $\sigma_{j}^{2}=k_{\sigma} \Delta_{j}^{2} ; \sigma_{j}$ - blur factor of fuzzy value $x_{j} ; k_{\sigma}=1 /(32 \ln 2)$.

The optimality criterion (8) is given as the sum of two quantities $R_{1}$ i $c \sum_{i=1}^{L} N_{i} G_{i, \min }$, where

$$
R_{1}=\sum_{i=1}^{L}\left(c N_{i} \pi_{\mathrm{G}, i} x_{\mathrm{G}, i}+c_{f} N_{i} E_{p, i}\right) .
$$

Since the first term in expression (10) is an additive function of the quantities $x_{G, i}, E_{p, i}$, and which have a Gaussian membership function [4], then $R_{1}$ will also have a Gaussian membership function [6]

$$
\mu\left(R_{1}\right)=\exp \left(-\left(R_{1}-R_{1,0}\right)^{2} /\left(2 \sigma_{R}^{2}\right)\right) \text {. }
$$

Based on the technological parameters $x_{j}, j=1, \ldots, 4$ are fuzzy quantities with the function (9), it was proved in [4] that

$$
\begin{gathered}
R_{\mathrm{l}, 0}=\sum_{i=1}^{L}\left(c N_{i} \pi_{\mathrm{G}, i} x_{\mathrm{G}, i}^{(0)}+c_{f} N_{i} m_{E, i}\right), \\
m_{E, i}=\sum_{j=0}^{M-1} a_{j} \prod_{k=1}^{m}\left(x_{0, k}^{(i)}\right)^{\varphi_{j k}}
\end{gathered}
$$

where $x_{0, k}^{(i)}$ - the modal values of the technological parameters, which are given in dimensionless form.

$$
\sigma_{R}^{2}=k \sum_{i=1}^{L}\left(\left(c N_{i} \pi_{\mathrm{G}, i}\right)^{2} \Delta_{\mathrm{G}, i}^{2}+\left(c_{f} N_{i}\right)^{2} \Lambda_{i}\right),
$$

where $x_{\mathrm{G}, i}^{(0)}$ - modal value of fuzzy quantity $x_{G, i}$; $\Lambda_{i}=\sum_{j=0}^{M-1} a_{j}^{2}\left(\sum_{r=1}^{m} \Delta_{r, i}^{2} \varphi_{j r}\left(x_{0, \mathrm{r}}^{(i)}\right)^{\varphi_{j r}-1} \prod_{\substack{q=1, q \neq i}}^{m}\left(x_{0, \mathrm{q}}^{(i)}\right)^{\varphi_{j q}}\right)$

Let $\gamma$ - be a slice of a fuzzy set defined by the membership function (11). Then $\mu\left(R_{1}\right)=\gamma$.

Given (11) and relations (12) - (14), we have

$$
\begin{aligned}
& \min : R(\bar{N})=c \sum_{i=1}^{L} N_{i}\left(\pi_{\mathrm{m}, i} x_{0, i}^{(m)}+\right. \\
& \left.+\frac{c_{f}}{c} \sum_{j=0}^{M-1} a_{j} \prod_{k=1}^{m}\left(x_{0, k}^{(i)}\right)^{\varphi_{j k}}+G_{i, \min }\right)+\sigma_{R_{1}} \sqrt{\ln \left(1 / \gamma^{2}\right)} .
\end{aligned}
$$

Optimization problem (15) with constraints (3) - (5) is a problem of discrete nonlinear programming and its solution can be found using the method of boundaries and branches [7].

Once the optimal number of units is determined, the task is to determine the optimal mode of operation of each GPU.

The task is to determine for each group of units, the number of which is determined by the criterion of optimality
(15), taking into account the constraints (3) - (5), the number of revolutions $n_{i}, i=\overline{1, m}$, so that the operating costs of each gas pumping unit would be minimal.

Therefore, the following should be minimized:

$$
R(\bar{n})=\sum_{i=1}^{m}\left(c G_{i}\left(n_{i}\right)+c_{f} E_{p, i}\left(n_{i}\right)\right) .
$$

The following restrictions on technological parameters must be fulfilled [5]:

$$
\begin{gathered}
T_{\text {out }}^{(i)} \leq T_{\text {out, } \max }^{(i)}, \\
T_{\text {tur }}^{(i)} \leq T_{\text {tur, } \max }^{(i)}, i=\overline{1, m},
\end{gathered}
$$

where $T_{\text {out }}^{(i)}$ - the gas temperature at the outlet of the supercharger; $T_{t u r}^{(i)}$ - he temperature of the combustion products at the outlet of the $i$-th low pressure turbine.

To prevent surges and to overload the supercharger, limit the lower $n_{i, \min }$ and upper $n_{i, \max }$ values of the rotational speed of the rotor

$$
n_{i, \text { min }} \leq n_{i} \leq n_{i, \text { max }} .
$$

It is established that the consumption of gas fuel $G_{i}$, the emission power $E_{p, i}$ оксидів азоту, of nitrogen oxides, the gas temperature at the outlet of the supercharger $T_{\text {out }}^{(i)}$ and the temperature of the combustion products at the outlet of the $i$-th turbine of low pressure $T_{\text {tur }}^{(i)}$ are functions of the regime parameters

$$
\begin{gathered}
G_{i}=f_{G}^{(i)}\left(n_{i}, P_{i n}, T_{i n}, \varepsilon, P_{c}, T_{c}\right), \\
E_{p, i}=f_{E, i}\left(n_{i}, P_{a c, i}, T_{a c, i}, T_{h t, i}\right), \\
T_{\text {out }}^{(i)}=f_{\text {out }}^{(i)}\left(n_{i}, P_{i n}, T_{i n}, \varepsilon, P_{c}, T_{c}\right), \\
T_{\text {tur }}^{(i)}=f_{\text {tur }}^{(i)}\left(n_{i}, P_{i n}, \varepsilon, P_{c}, T_{c}\right) .
\end{gathered}
$$

Expressions (20) - (23) have the following notation: $P_{i n}$, $T_{i n}$ - gas pressure and temperature at the inlet of the centrifugal supercharger; $P_{c}, T_{c}$ - pressure and ambient temperature; $P_{a c, i}, T_{a c, i}$ - gas pressure and temperature at the outlet of the $i$-th axial compressor; $T_{h t, i}$ - is the outlet temperature of the high pressure turbine.

The degree of pressure increase $\varepsilon$ is determined by the following formula [8]: $\varepsilon=P_{a, \text { out }} / P_{a, \text { in }}$, where $P_{a, \text { in }}, P_{a, \text { out }}$ - are absolute pressures at the inlet and outlet of the supercharger.

Since centrifugal superchargers $T_{\text {out }}^{(i)}$ operate in parallel to the common manifold, the $P_{\text {in }}$ pressure and $T_{i n}$ temperature at the inlet of the supercharger, the $P_{\text {out }}$ pressure at the outlet of the supercharger, the pressure $P_{c}$ and the ambient temperature $T_{c}$ are assumed to be the same. 
The functional dependencies (20) - (23) are approximated by a polynomial dependence similar to (6). For this purpose, a method based on the ideas of genetic algorithms was used [8].

For a certain mode of operation of the centrifugal supercharge $n_{i}$ the technological parameters $P_{i n}, T_{i n}, P_{c}, T_{c}$ and $\varepsilon$, which are arguments of functional dependencies (22) and (23), will take specific values. Then dependencies (22) and (23) will be functions of only one variable

$$
\begin{aligned}
& T_{\text {out }}^{(i)}=\theta_{\text {out }}^{(i)}\left(n_{i}\right), \\
& T_{\text {tur }}^{(i)}=\theta_{\text {tur }}^{(i)}\left(n_{i}\right) .
\end{aligned}
$$

For values of $T_{\text {out,max }}^{(i)}$ and $T_{\text {tur, } \text { max }}^{(i)}$, яwhich are defined by restrictions (16) and (17), relations (24) and (25) will be as follows: $T_{\text {out }, \text { max }}^{(i)}=\theta_{\text {out }}^{(i)}\left(n_{i}\right), T_{\text {tur }, \text { max }}^{(i)}=\theta_{\text {tur }}^{(i)}\left(n_{i}\right), i=\overline{1, m}$.

Solving the obtained equations with respect to ni gives the following values: $n_{\text {out, } \max }^{(i)}$ and $n_{\text {tur, } \max }^{(i)}$. Then the upper limit for the number of revolutions of the rotor of the supercharger in restriction (19) is determined by the following condition:

$$
\tilde{n}_{i, \max }=\min :\left\{n_{\text {out }, \text { max }}^{(i)}, n_{\text {tur }, \text { max }}^{(i)}, n_{i, \max }\right\}, i=\overline{1, m} .
$$

Thus, taking into account (26), we modify condition (19)

$$
n_{i, \min } \leq n_{i} \leq \tilde{n}_{i, \max }, i=\overline{1, m}
$$

Compliance with condition (27) is a guarantee that constraints (17) and (18) will be satisfied.

In addition, it is necessary to take into account the additional condition that stipulates the provision of a given balance of pumping of natural gas

$$
\sum_{i=1}^{m} k_{i} q_{i}\left(n_{i}\right)=q_{0},
$$

where $q=Q_{0} / m$ - average supercharger performance, which is driven by task $Q_{0}$ for gas pumping.

The coefficient of $k_{i}$ load of the $i$-th supercharger in the group of parallel-working $m$ superchargers is defined by a formula similar to (1).

The performance of the $q_{i}$ supercharger is a function of technological parameters such as $P_{i n}, T_{i n}, \varepsilon, P_{c}$ and $T_{c}$, which for a particular mode of operation of the GPU take certain specific values. Therefore, in restriction (28) $q_{i}=q_{i}\left(n_{i}\right)$.

For a number of reasons, the coefficients of polynomial models of type (6) by are calculated with some inaccuracy. These reasons include: errors in the methods of indirect measurement of fuel gas flow and supercharger performance by pressure drop, interpolation error in determining atmospheric pressure and nitrogen oxide emission power. The need for interpolation is due to the multi-step steps of discreteness $P_{i n}, T_{i n}, T_{\text {out }}$ and $T_{c}$, on the one hand, and the atmospheric pressure $P_{c}$ i and the emission power $E_{p}$, on the other. For example, measuring the performance of a supercharger over a pressure drop on a confuser can cause measurement errors of up to $20 \%$ [9]. In addition, the process of compression of natural gas is under the influence of various external influences, which distorts the measurement results of technological parameters. All this gives grounds to consider the coefficients of empirical models as fuzzy quantities.

The results of observations on the capacity of the emissions of nitrogen oxides into the environment and the functional dependence $q_{i}=f_{q, i}\left(n_{i}, P_{i n}, T_{i n}, \varepsilon, P_{c}, T_{c}\right), \quad i=\overline{1, m}$ are approximated by polynomials similar to (6)

$$
\begin{gathered}
E_{p, j}\left(\overline{\mathrm{a}}_{E}^{(j)}, \overline{\mathrm{x}}_{E}\right)=\sum_{i=0}^{M-1} a_{E, i}^{(j)} \prod_{k=1}^{r_{1}} x_{E, k}^{\varphi_{k i}}, \\
q_{j}\left(\overline{\mathrm{a}}_{q}, \overline{\mathrm{x}}_{q}\right)=\sum_{i=0}^{M-1} a_{\mathrm{q}, i}^{(j)} \prod_{k=1}^{r_{2}} x_{q, k}^{\varphi_{k i}}, \quad j=\overline{1, m},
\end{gathered}
$$

where $\bar{x}_{E}$ i $\bar{x}_{q}$ - vectors of technological parameters whose components are drawn to dimensionless units by the formula (7); for the empirical model (29): $X_{1}=n_{j}, X_{2}=P_{a c, j}, X_{3}=T_{a c, j}$ and $X_{4}=T_{h t, j}$; the empirical model (30): $X_{1}=n_{j}, X_{2}=P_{i n}$, $X_{3}=T_{i n}, X_{4}=\varepsilon, X_{5}=P_{c}$, and $X_{6}=T_{c} ; r_{1}$ and $r_{2}$ - vector dimensions $\bar{x}_{E}$ i $\bar{x}_{q}$.

Synthesis of empirical models (29) and (30) is carried out by using the method [8], which is based on the theory of genetic algorithms. The result is an empirical model of optimal complexity, in which the number of coefficients of the non-zero model is less than $M$. Other coefficients of the empirical models assume zero values.

Each mode of operation of the $i$-th supercharger is characterized by a certain set of technological parameters. The values of each of these technological parameters, in addition to the rotational speed of the rotor of the supercharger, which are reduced to dimensionless units, can be substituted into the expressions of the synthesized empirical models (29) and (30). As a result, we obtain polynomials

$$
\begin{gathered}
\tilde{E}_{p, j}\left(x_{1}, \bar{A}_{E}^{(j)}\right)=\sum_{k=0}^{n_{E, j}} A_{E, k}^{(j)} x_{1}^{k}, \\
\tilde{q}_{j}\left(x_{1}, \bar{A}_{q}^{(j)}\right)=\sum_{k=0}^{n_{q, j}} A_{q, k}^{(j)} x_{1}^{k}, j=\overline{1, m},
\end{gathered}
$$

where $n_{E, j} \leq r_{1}$ and $n_{q, j} \leq r_{2}$.

It is assumed that the coefficients of the polynomials of models (31) and (32) are fuzzy quantities with a triangular membership function approximated by a Gaussian function similar to (9). For the coefficients of polynomials (31) and (32)

$$
\mu\left(A_{E, k}^{(j)}\right)=\exp \left(-\left(A_{E, k}^{(j)}-A_{E_{0}, k}^{(j)}\right)^{2} /\left(2\left(\sigma_{E, k}^{(j)}\right)^{2}\right)\right)
$$




$$
\begin{aligned}
& \mu\left(A_{q, k}^{(j)}\right)=\exp \left(-\left(A_{q, k}^{(j)}-A_{q_{0}, k}^{(j)}\right)^{2} / 2\left(\sigma_{q, k}^{(j)}\right)^{2}\right), \\
& j=\overline{1, m}
\end{aligned}
$$

where $A_{E_{0}, k}^{(j)}, A_{q_{0}, k}^{(j)}$ - modal values of membership functions (33) and (34).

Since the triangular functions are fuzzy quantities $A_{E, k}^{(j)}$ and $A_{q, k}^{(j)}$ approximated by a Gaussian function, then $\left(\sigma_{E, k}^{(j)}\right)^{2}=k_{\sigma}\left(\Delta_{E, k}^{(j)}\right)^{2}$ and $\left(\sigma_{q, k}^{(j)}\right)^{2}=k_{\sigma}\left(\Delta_{q, k}^{(j)}\right)^{2}$.

The magnitude of the uncertainty intervals $\Delta_{E, k}^{(j)}$ and $\Delta_{\mathrm{q}, k}^{(j)}$ fuzzy values $A_{E, k}^{(j)}$ i $A_{q, k}^{(j)}$ define as follows: $\Delta_{E, k}^{(j)}=\delta_{E, k}^{(j)} A_{E_{0}, k}^{(j)} \quad$ and $\quad \Delta_{q, k}^{(j)}=\delta_{q, k}^{(j)} A_{q_{0}, k}^{(j)} \quad$, where $\left\{\delta_{E, k}^{(j)}, \delta_{q, k}^{(j)}\right\} \in(0 ; 1]$.

Fuel gas consumption is measured with an error not exceeding 2\% [10]. Therefore, the approximation of (20) is a polynomial

$$
G_{j}\left(\overline{\mathrm{a}}_{G}, \overline{\mathrm{x}}_{G}\right)=\sum_{i=0}^{M-1} a_{\mathrm{G}, i}^{(j)} \prod_{k=1}^{r_{2}} x_{\mathrm{G}, k}^{\varphi_{k i}}, \quad j=\overline{1, m},
$$

assumes that the coefficients $a_{\mathrm{G}, i}^{(j)}$ of the polynomial (35) are clear numbers.

Based on the assumptions made, we formulate the problem of optimal control of a group of parallel switched GPUs to determine the rotor speed of each supercharger, so that the criterion of optimality

$$
\begin{aligned}
& R\left(\bar{x}^{(1)}\right)=c \sum_{j=1}^{m}\left(\sum_{k=0}^{n_{G, j}} A_{\mathrm{G}, k}^{(j)} x_{1, j}^{k}+r_{c} \sum_{k=0}^{n_{E, j}} A_{E_{0}, k}^{(j)} x_{1, j}^{k}\right. \\
& \left.+K_{E}\left(\sum_{k=0}^{n_{E, j}}\left(\delta_{E, k}^{(j)} A_{E_{0}, k}^{(j)}\right)^{2} x_{1, j}^{2 k}\right)^{1 / 2}\right)
\end{aligned}
$$

has acquired a minimum value under the following restrictions:

$$
\begin{aligned}
& \sum_{j=1}^{m} k_{j}\left(\sum_{k=0}^{n_{q, j}} A_{q_{0}, k}^{(j)} x_{1, j}{ }^{k}+\right. \\
& \left.+K_{q}\left(\sum_{k=0}^{n_{q, j}}\left(\delta_{q, k}^{(j)} A_{q_{0}, k}^{(j)}\right)^{2} x_{1, j}^{2 k}\right)^{1 / 2}\right)=q_{0}, \\
& 0 \leq x_{1, j} \leq \beta_{j}, \quad j=\overline{1, m},
\end{aligned}
$$

where $x_{1, j}$ - the number of revolutions of the rotor $j$ supercharger in dimensionless units; $K_{q}=k_{\sigma} \sqrt{\ln \left(1 / \gamma^{2}\right)}$; $0<\delta_{\mathrm{q}, k}^{(j)} \leq 1 ; \quad A_{q_{0}, k}^{(j)}$ - modal values of polynomial coefficients; $\beta_{j}=\tilde{n}_{j, \max }-n_{j, \min } / \Delta_{n}^{(j)}, j=\overline{1, m} ; \Delta_{n}^{(j)}=n_{j, \text { max }}-n_{j, \min }$.

Formed problem (36) - (38) of optimal control of work of parallel working GPA is a problem of nonlinear programming and for finding of its solution it is possible to apply, for example, algorithm of internal points [11] is implemented in software MatLab (built-in function fmincon).

The result of implementing the algorithm for solving problems (36) - (38) is the optimum value of the rotor rotor speed in dimensionless units. The transition to dimensional units is made by the formula $n_{j}=\Delta_{n, j} x_{1, j}+n_{j, \min }, j=\overline{1, m}$.

The obtained values of $\mathrm{nj}$ minimize the optimality criterion (36), taking into account the constraints (37) and (38).

Checking the efficiency of the developed algorithms was carried out on the industrial data obtained in the conditions of operation of the compressor station of Bogorodchansk linear control of the main pipelines of JSC "PrykarpatTransgaz". The following initial data were obtained:

A. to determine the optimal number of concurrent units in the group:

- the number of superchargers between groups is distributed as follows: first group - 3; second group 7; third group - 4;

- the total performance of the superchargers for each of the three groups is: for the first group - 1782000 $\mathrm{m}^{3} / \mathrm{h}$; for the second group $-2749300 \mathrm{~m}^{3} / \mathrm{h}$; for the third group - $2371680 \mathrm{~m}^{3} / \mathrm{h}$;

- specified volume for gas transportation is $3700800 \mathrm{~m}^{3} / \mathrm{h}$;

- the cost of fuel gas is $10226 \mathrm{UAH} / \mathrm{m}^{3}$;

- environmental tax on nitrogen oxide emissions 2451,84 $\mathrm{UAH} / \mathrm{m}^{3}$;

- emissions of nitrogen oxides for all groups of GPA were $G_{i, \min }=2,88 \mathrm{~kg} / \mathrm{s} ; \pi_{m, i}=1,4327 \mathrm{~kg} / \mathrm{s}, \forall i$;

- generalized coefficients of technical condition for each group of superchargers: the first group - 0,916; second group - 0,920 ; the third group is 0.953 .

B. to determine the optimum speed of each rotor supercharger in the group:

- $\quad$ the degree of pressure increase - 1,42;

- $\quad$ pressure at the inlet of the supercharger - 4,.7 MPa;

- ambient temperature - 16 degrees $\mathrm{C}$;

- $\quad$ ambient pressure - $749 \mathrm{mmHg}$;

- $\quad$ planned task for gas pumping - $3700800 \mathrm{~m}^{3} / \mathrm{h}$;

- the gas temperature at the inlet of the supercharger is not more than +60 degrees $C$;

- $\quad$ exhaust gas temperature not more than +700 degrees C.

The calculations made on the developed computer program gave the following result: 
- $\quad$ number of supercharges: in the first group - $N_{1}^{*}=3$; in the second group $-N_{2}^{*}=0$; in the third group $N_{3}^{*}=4$;

- cost of the compressor station operation $R\left(\bar{N}^{*}\right)=274,85 U A H / s$;

- the imbalance value $53,26 \mathrm{~m}^{3} / \mathrm{s}$. This means that one of the centrifugal natural gas superchargers is underexposed by $53,26 \mathrm{~m}^{3} / \mathrm{s}$;

- the rotor speed of the first group supercharger - the first supercharger - $4300 \mathrm{rpm}$; the second supercharger - $4805 \mathrm{rpm}$; the third supercharger $5105 \mathrm{rpm}$;

- the number of rotations of the rotor of the superchargers of the second group - the first supercharger - $4585 \mathrm{rpm}$; the second supercharger $4730 \mathrm{rpm}$; the third supercharger - $4450 \mathrm{rpm}$;

- the number of revolutions of the rotor of the superchargers of the third group - the first supercharger - $5020 \mathrm{rpm}$; the second supercharger $4920 \mathrm{rpm}$; the third supercharger - $4250 \mathrm{rpm}$.

Taking into account the current state of the superchargers allows to save resources in the amount of $21,42 U A H / h$.

\section{CONCLUSIONS}

The proposed system of optimal control of the GPU solves two problems - the optimal distribution of the planned amount of natural gas between the parallel operating units and the determination of the optimum number of rotations of the rotor of the supercharger. The cost of fuel gas, taking into account the capacity of the nitrogen oxide into the environment, is selected as the criterion of optimality, which is the main difference between the work and the work where similar tasks were considered.

The peculiarity of the work is the fact that the formalization of the tasks of optimal control of the GPU operation takes into account the uncertainty inherent in the process of compression of natural gas. For this purpose, the apparatus of fuzzy algebra was used, which made it possible to take into account the inaccuracies that arise as a result of measuring technological parameters and as a result of processing the obtained data on the technological process.

\section{REFERENCES}

[1] A. S. Dovbysh, A. S.Dovbysh, I. V. Shelekhov, and I. V. Shelehov, Fundamentals of pattern recognition theory. Sums: SSU, 2015. [in Ukrainian].

[2] O. Mirzoyeva "Analysis of existing methods, models, technical means to monitor emissions of harmful substances from the combustion chamber", Transactions of Kremenchuk Mykhailo Ostrohradskyi National University, vol. 3 (104). pp. 100 - 105, 2017. [in Ukrainian].

[3] M. I. Horbiychuk, O. T. Bila, and N.T. Lazoriv, "Optimal distribution of parallel working equipment with regard to environmental taxes under understanding conditions", Scientific Bulletin of IvanoFrankivsk National Technical University of Oil and Gas, vol. 1 (46), 35-45, Jun. 2019. [in Ukrainian].

[4] M. Gorbiychuk, B. Pashkovskyi, O. Moyseenko, and N Sabat, "Solution of the optimization problem on the control over operation of gas pumping units under fuzzy conditions", Eastern-European Journal of Enterprise Technologies, vol. 5, no. 2 (89), pp. 65-71. Sep. 2017. doi:10.15587/1729-4061.2017.111349.

[5] L. G. Raskin, and O. V. Seraya, Nechetkaya matematika [Fuzzy math]. Kharkiv: Parus, 2008. [in Russian].

[6] I. V. Sergienko, O. O. Iemets, and O. O. Yemets, "Optimization Problems with Interval Uncertainty: Branch and Bound Method", Cybernetics and Systems Analysis, Cybernetics and Systems Analysis, vol. 49, no. 5, pp. 673-683, Sep. 2013. doi:10.1007/s10559013-9554-8.

[7] M. I. Gorbiychuk, V. M. Medvedchuk, and A. N. Lazoriv, "Analysis of parallel algorithm of empirical models synthesis on principles of genetic algorithms", Journal of Automation and Information Sciences, vol. 48 (2), pp. 54-73, Jan. 2016 doi:10.1615/JAutomatInfScien.v48.i2.60

[8] Ilchenko B.S. Diagnosis of functional and technical state of gaspumping units: monograph. Kharkiv: Hark. nat. Acad. of city mastering KNAMG, 2011. [in Ukrainian].

[9] SOU 60.3-30019801-011: Compressor stations. Control of thermal and environmental characteristics of gas-pumping units: [Effective from. 12/22/2004], K.: DK Ukrtransgaz, 2004. [in Ukrainian].

[10] R. Byrd, J. Gilbert, and J. Nocedal, "A trust region method based on interior point techniques for nonlinear programming", Mathematical programming, vol. 89 (1), pp. 149-185, Nov. 2000. doi:10.1007/PL00011391. 